\title{
Role of feline sarcoma-related protein in the viability and apoptosis of bladder cancer cells
}

\author{
XUDONG HU $^{1}$, ZHIWEN GUO ${ }^{1}$, JIFEI XU ${ }^{1}$, XIANGBAO MEI ${ }^{1}$, \\ MANHUA BI ${ }^{1}$, FANG JIANG ${ }^{1}$, DEXIN YU ${ }^{2}$ and CAIYUN ZHONG ${ }^{3}$ \\ ${ }^{1}$ Department of Urology, Anqing First People's Hospital, Anqing, Anhui 246000; ${ }^{2}$ Department of Urology, \\ The Second Hospital of Anhui Medical University, Hefei, Anhui 230032; ${ }^{3}$ Department of Nutrition and Food Safety, \\ School of Public Health, Nanjing Medical University, Nanjing, Jiangsu 211166, P.R. China
}

Received December 24, 2017; Accepted November 19, 2018

DOI: $10.3892 / \mathrm{mmr} .2019 .10204$

\begin{abstract}
Feline sarcoma-related protein (Fer) is a type of nuclear and cytoplasmic non-receptor protein tyrosine kinase, which is associated with the progression of numerous types of cancer. Previously, we identified that Fer is associated with the migration and invasion of bladder cancer. The present study aimed to investigate the role of Fer in bladder cancer cell viability and apoptosis. Reverse transcription-quantitative polymerase chain reaction and western blot analysis were performed to detect the expression levels of Fer; short interference RNA (siRNA) and overexpression vectors were used to downregulate or upregulate Fer expression, respectively. The effects on cell proliferation ability and cell apoptosis were then tested by MTT assay and flow cytometry. The results revealed that Fer expression was upregulated in bladder cancer cell lines. Downregulation of Fer expression by siRNA significantly suppressed T24 cell viability and induced apoptosis, as well as inducing cell cycle arrest. Conversely, Fer overexpression in 5637 cells significantly promoted cell viability and cell cycle progression, but inhibited cell apoptosis. Furthermore, the suppression and overexpression of Fer significantly altered the expression of cleaved caspase- 3 and $\mathrm{Bcl}-2$, and dysregulated the P38 mitogen-activated protein kinase signaling pathway. The findings of the present study indicate a possible molecular mechanism of Fer in bladder cancer and may be considered as a potential target in the treatment of this disease.
\end{abstract}

Correspondence to: Professor Caiyun Zhong, Department of Nutrition and Food Safety, School of Public Health, Nanjing Medical University, 818 Tianyuan Road, Nanjing, Jiangsu 211166, P.R. China E-mail: cyzhong@njmu.edu.cn

Dr Dexin Yu, Department of Urology, The Second Hospital of Anhui Medical University, 678 Furong Road, Hefei, Anhui 230032, P.R. China

E-mail: yudx_urology@126.com

Key words: feline sarcoma-related protein, bladder cancer, proliferation, apoptosis

\section{Introduction}

Bladder cancer is one of the most common malignant tumors of the genitourinary system worldwide (1). Researchers have identified that $75-85 \%$ of patients with bladder cancer have non-muscle-invasive bladder cancer (NMIBC), while $15-25 \%$ of cases progressed to muscle-invasive bladder cancer (MIBC) (1). At present, radical cystectomy with urinary diversion is the standard treatment for patients with NMIBC and MIBC (2); however, $50 \%$ of patients with MIBC develop metastatic disease, which is likely to be fatal. The prognosis following recurrence after cystectomy is poor (3). Therefore, in order to gain a comprehensive understanding of the pathogenesis of bladder cancer, the molecular mechanisms underlying the occurrence and development of this disease must be identified. This may provide insight into novel and effective treatment strategies for the treatment of bladder cancer.

Feline sarcoma-related protein (Fer) is a unique Src homology 2 non-receptor tyrosine kinase, which is expressed in certain mammalian cell subpopulations, and resides in the cytoplasm and nucleus (4). Fer is highly expressed in numerous types of cancer, including lung (5), hepatic (6), prostate (7), breast (8) and bladder cancer (9). Previous studies have demonstrated that Fer expression is associated with the proliferation of certain cancer cell lines cultures; the poor prognosis of cancer has been associated with increased Fer expression levels (10-12). In addition, Fer is involved in the signaling downstream of the receptor systems of cell proliferation and invasion in several cell types (13). These studies indicate a potential function of Fer in the progression of cancer; however, the exact roles and underlying mechanisms of Fer in the proliferation and apoptosis of bladder cancer remain to be fully elucidated. To the best of our knowledge, the present study is the first to determine the role of Fer in the viability and apoptosis of bladder cancer cells.

In the present study, the effects of transfection of bladder cancer cells with short interfering RNA against Fer (Fer-siRNA) and Fer overexpression vector (Vector-Fer) on the expression of Fer mRNA and protein. Furthermore, the underlying mechanism of Fer in the proliferation and apoptosis of bladder cancer cells was investigated. The results of this study demonstrated that Fer serves a role in development of the bladder cancer. 


\section{Materials and methods}

Cell line culture and maintenance. Bladder cancer cell lines T24, 5637 and an immortalized normal human epithelial cell line SV-HUC-1 were purchased from the Cell Bank of Type Culture Collection of Chinese Academy of Sciences (Shanghai, China). T24 and 5637 cell lines were cultured in RPMI-1640 (Gibco; Thermo Fisher Scientific, Inc., Waltham, MA, USA) with $10 \%$ fetal bovine serum (FBS; HyClone; GE Healthcare Life Sciences, Logan, UT, USA) at $37^{\circ} \mathrm{C}$ under $5 \% \mathrm{CO}_{2}$ and $95 \%$ humidified air. SV-HUC-1 cells were cultured under an atmosphere of $5 \% \mathrm{CO}_{2}$ at $37^{\circ} \mathrm{C}$ in $\mathrm{F} 12 \mathrm{k}$ medium (F12K; WISENT Inc., Saint-Jean-Baptiste, QC, Canada) with $10 \%$ fetal bovine serum (PAA Laboratories; GE Healthcare, Chicago, IL, USA), $100 \mathrm{U} / \mathrm{ml}$ penicillin, and $100 \mathrm{lg} / \mathrm{ml}$ streptomycin.

Reverse transcription-quantitative polymerase chain reaction $(R T-q P C R)$. Total RNA was isolated from T24, 5637 and SV-HUC-1 cells $\left(2 \times 10^{6}\right.$ cells/ml) by RNAiso Plus (Takara Bio, Inc., Otsu, Japan), according to the manufacturer's protocols, and $5 \mu \mathrm{g}$ of each sample was reverse-transcribed using the M-MLV First-strand Synthesis System (Promega Corporation, Madison, WI, USA) as follows: $37^{\circ} \mathrm{C}$ for $25 \mathrm{~min}$, followed by incubation at $85^{\circ} \mathrm{C}$ for $5 \mathrm{sec}$ in $20 \mu \mathrm{l}$ of reaction volume. All reactions were performed in triplicate using the MJ Real-Time PCR System (Bio-Rad Laboratories, Inc., Hercules, CA, USA). qPCR was performed using the Power SYBR Green Master Mix (Takara Bio, Inc.) and an ABI 7300 real-time PCR detection system (Applied Biosystems; Thermo Fisher Scientific, Inc.), using the following primers (all primers were synthesized by Invitrogen; Thermo Fisher Scientific, Inc.): Fer, forward 5'-TTCGAGGGCACTGGGTTTTC-3', reverse 5'-TTCCCT TGCCCAGTAATTCTCC-3'; GAPDH, forward 5'-GGTGAA GGTCGGAGTCAACGGA-3', reverse 5'-GAGGGATCTCGC TCCTGGAAGA-3'; GAPDH served as an internal control. qPCR was performed under the following thermocycling conditions: $96^{\circ} \mathrm{C}$ for $2 \mathrm{~min}$; followed by 21 cycles of $96^{\circ} \mathrm{C}$ for $30 \mathrm{sec}, 55^{\circ} \mathrm{C}$ for $30 \mathrm{sec}, 68^{\circ} \mathrm{C}$ for $30 \mathrm{sec}$, and a final elongation at $68^{\circ} \mathrm{C}$ for $30 \mathrm{sec}$. The relative levels of individual mRNA in each sample were normalized to GAPDH and calculated using the $2^{-\Delta \Delta \mathrm{Cq}}$ method (14).

Western blotting. Cells $\left(5 \times 10^{7}\right.$ cells $\left./ \mathrm{ml}\right)$ were harvested $72 \mathrm{~h}$ following infection and lysed in Radioimmunoprecipitation Assay buffer (Fermentas; Thermo Fisher Scientific, Inc.) supplemented with $1 \%$ protease inhibitors (Sigma-Aldrich; Merck KGaA, Darmstadt, Germany) on ice, followed by centrifugation at $12,000 \mathrm{x}$ g for $15 \mathrm{~min}$ at $4^{\circ} \mathrm{C}$. Protein concentrations were measured with the Bicinchoninic Protein Assay (Pierce; Thermo Fisher Scientific, Inc.). Subsequently, proteins were diluted to equal concentrations (20 or $30 \mathrm{mg}$ ), boiled for $5 \mathrm{~min}$ and separated by 7.5-10\% SDS-PAGE, followed by transblotting to an Immun-Blot polyvinylidene difluoride membrane (Bio-Rad Laboratories, Inc.). The membranes were blocked with $5 \%$ defatted milk in TBS $+0.1 \%$ Tween-20 and probed with primary antibodies overnight at $4^{\circ} \mathrm{C}$. Membranes were subsequently incubated with horseradish peroxidase (HRP)-conjugated secondary antibody for $1 \mathrm{~h}$ at room temperature. Protein bands were visualized using an
Enhanced Chemiluminescence Detection kit (GE Healthcare). The following rabbit monoclonal antibodies were used: Anti-Fer (1:500; catalog no. ab191060; Abcam, Cambridge, UK); anti-Bcl-2 monoclonal antibody [1:500; catalog no. 4223; Cell Signaling Technology (CST), Inc., Danvers, MA, USA]; CyclinD1 (1:500; catalog no. 2978; CST); p21(1:500; catalog no. 2947; CST); cleaved Caspase-3 (1:500; catalog no. 9661; CST); phosphorylated (p)-p38 MAPK (Thr180/Tyr182) (1:500; catalog no. 4511; CST); Rabbit anti-GAPDH polyclonal antibody (1:500; catalog no. Ab9485; Abcam) was used as an internal control protein. Densitometric analysis to quantify protein expression levels was performed using Image J software v1.46 (National Institutes of Health, Bethesda, MD, USA).

Small interfering RNA (siRNA) transfection. siRNAs targeting Fer and a negative control (NC) siRNA were purchased from Shanghai GenePharma Co., Ltd. (Shanghai, China). The targeting sequences of three Fer-siRNAs (siRNA1, 5'-AAA GAAATTTATGGCCCTGAG-3'; siRNA2, 5'-CAGATAGAT CCTAGTACAGAA-3'; siRNA3, 5'-AACTACGGTTGCTGG AGACAG-3') and one NC-siRNA (5'-UUCUCCGAACGU GUCACGU-3') were designed using an RNAi algorithm available online at The RNAi Web (http://www.rnaiweb.com). For transfection, the siRNAs $(100 \mathrm{nmol} / \mathrm{l})$ were transfected into T24 using Lipofectamine ${ }^{\circledR} 2000$ transfection reagent (Invitrogen; Thermo Fisher Scientific, Inc.), according to the manufacturer's protocols. T24 cells $\left(4 \times 10^{5}\right.$ cells $\left./ \mathrm{cm}^{2}\right)$ were grown in regular medium for $72 \mathrm{~h}$ at $37^{\circ} \mathrm{C}$ and subsequently transfected with siRNAs, control cells were transfected with the NC siRNA. Cells were transfected at $37^{\circ} \mathrm{C}$ for 48 and $72 \mathrm{~h}$ at which time they were harvested for RNA and protein extraction, respectively.

Generation of plasmid constructs and establishment of Fer overexpression cell lines. To generate Fer overexpression vectors, Fer coding sequences were obtained by RT-PCR (primers: Forward 5'-TTCGAGGGCACTGGGTTTTC-3', reverse 5'-TTCCCTTGCCCAGTAATTCTCC-3') and cloned into the pEGFP-N1 vector (Clontech Laboratories, Inc., Mountainview, CA, USA). The resulting plasmid was designated as pEGFP-N1-Fer and was transfected into 5637 bladder cancer cells to induce Fer overexpression, 5637 cells were plated in regular medium at a density of $1 \times 10^{4}$ cells $/ \mathrm{cm}^{2}$ for $72 \mathrm{~h}$ at $37^{\circ} \mathrm{C}$ and then transfected with pEGFP-N1-Fer; pEGFP-N1 empty vector was used as a control, and the resulting cell lines were designated as 5637/pEGFP-N1-Fer and 5637/pEGFP-N1, respectively. After $24 \mathrm{~h}$ post-transfection, G418 solution was added to cells for the selection of stable clones, which were then cultured in medium containing G418. Both 5637/pEGFP-N1-Fer and 5637/pEGFP-N1 cells were maintained in fresh regular medium for 2 days and then harvested for cell number counting.

MTT assay. Bladder cancer cells were seeded $\left(5 \times 10^{3}\right.$ cells/well) in flat-bottomed 96-well plates. After $24 \mathrm{~h}$, cells were transfected for 1, 2, 3 or 4 days, as aforementioned. Following culture, $10 \mu 1 \mathrm{MTT}(5 \mathrm{mg} / \mathrm{ml})$ was added to each well and plates were incubated at $37^{\circ} \mathrm{C}$ for $4 \mathrm{~h}$. The medium was removed and $100 \mu \mathrm{l}$ dimethyl sulfoxide solution was added to each well to dissolve the purple formazan crystals. Absorbance was 
A

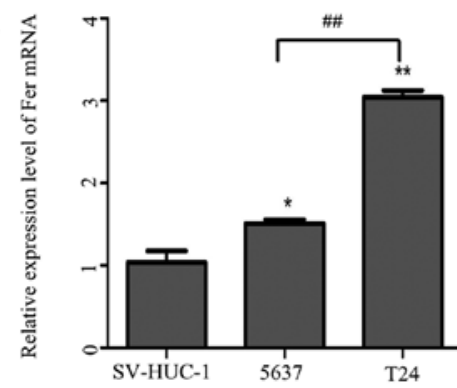

C

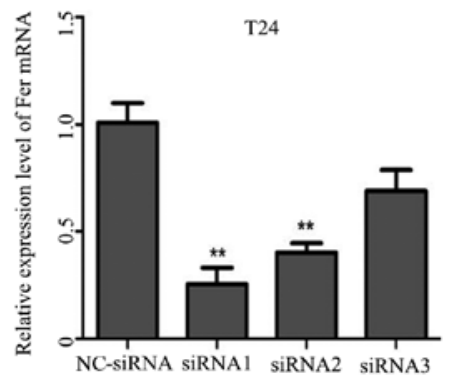

E

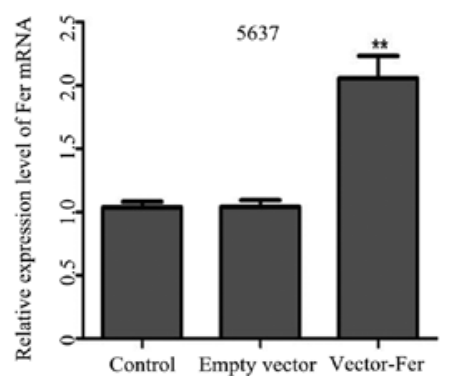

B

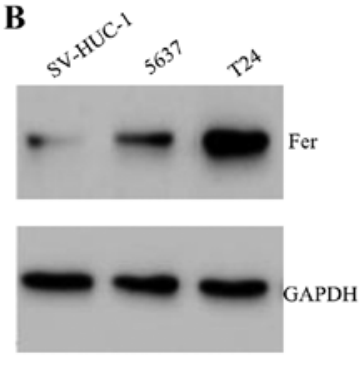

D

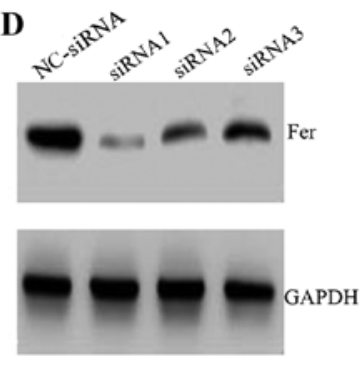

F

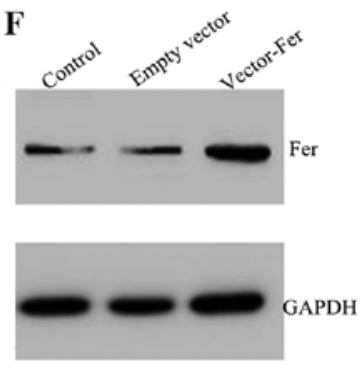

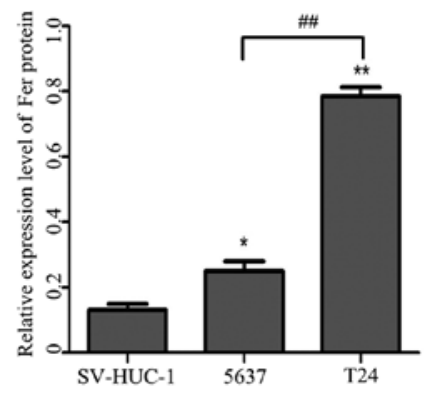
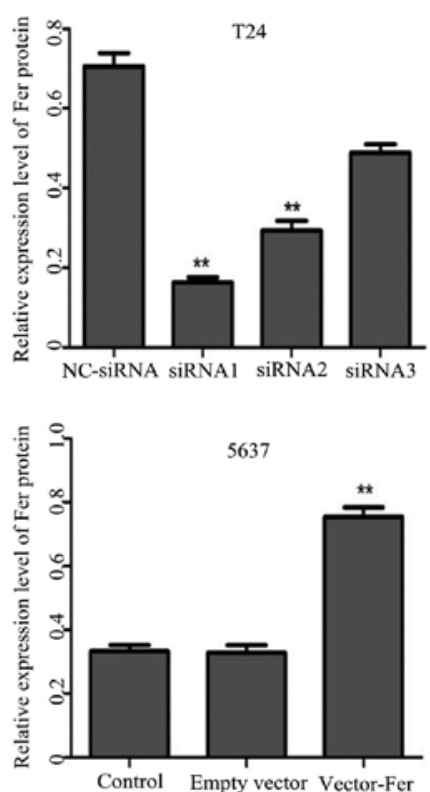

Figure 1. Efficacy of Fer-siRNA and vector overexpression of Fer in bladder cancer cells. (A and B) Fer mRNA and protein expression levels were higher in two bladder cancer cell lines (5637 and T24) compared with in the normal bladder epithelium cell line SV-HUC-1, as evaluated by RT-qPCR and western blot analysis, respectively. GAPDH was used as an internal control. Data are presented the mean \pm standard deviation; $\mathrm{n}=3$; ${ }^{*} \mathrm{P}<0.05$, $5637 \mathrm{vs.} \mathrm{SV-HUC-1} \mathrm{cells;}$ ${ }^{* *} \mathrm{P}<0.01$, T2 4 vs. SV-HUC-1 cells; ${ }^{\# \#} \mathrm{P}<0.01$, T24 vs. 5637). (C and D) Relative Fer mRNA relative levels in the Fer-siRNA1 and Fer-siRNA2 groups were significantly decreased compared with the NC-siRNA group, as evaluated by RT-qPCR. Similar results were obtained for protein expression as determined by western blot analysis. Data are presented as the mean \pm standard deviation; $n=3 ;{ }^{* *} \mathrm{P}<0.01$ vs. NC-siRNA. (E and F) Fer mRNA and protein expression was upregulated by $\sim 2$-fold in the Vector-Fer group. Data are presented as the mean \pm standard deviation; $n=3$; ${ }^{* *} \mathrm{P}<0.01$ vs. Empty vector and Control. Fer, feline sarcoma-related protein; NC, negative control; RT-qPCR, reverse transcription-quantitative polymerase chain reaction; siRNA, small interfering RNA; Vector-Fer, Fer overexpression plasmid.

measured at $490 \mathrm{~nm}$ using a microplate reader to determine cell viability; three replicate wells were analyzed per assay and each experiment was repeated three times.

Cell cycle and apoptosis assays. Transfected cells $\left(3 \times 10^{5}\right.$ cells/well) were harvested using $0.25 \%$ trypsin at $37^{\circ} \mathrm{C}$ for $30 \mathrm{~min}$, and were subsequently added to $1 \mathrm{ml}$ of $70 \%$ cold ethanol overnight at $4^{\circ} \mathrm{C}$. The next day, cells were centrifuged at $12,000 \mathrm{x} \mathrm{g}$ for $5 \mathrm{~min}$ at room temperature, treated with $100 \mu \mathrm{l}$ RNase at $37^{\circ} \mathrm{C}$ for $30 \mathrm{~min}$, and stained with $400 \mu \mathrm{l}$ propidium iodide (PI; catalog no. KGA511; Nanjing KeyGen Biotech, Co., Ltd., Nanjing, China) at $4^{\circ} \mathrm{C}$ for $30 \mathrm{~min}$. The percentage of cells in G0/G1, S and G2/M phase was determined by DNA flow cytometry with Cell Quest software v5.1 (BD Biosciences, San Jose, CA, USA).

Annexin V-fluorescein isothiocyanate (FITC)/PI Apoptosis Detection kit (catalog no. KGA108; Nanjing KeyGen Biotech Co., Ltd.) was used to analyze apoptosis; culture medium without FBS was added to the cells which had been transfected at $37^{\circ} \mathrm{C}$, followed by further culture for $24 \mathrm{~h}$. Cells were harvested and centrifuged at $12,000 \mathrm{x} \mathrm{g}$ for $5 \mathrm{~min}$ at room temperature. The medium was removed, and cells were washed once in PBS. Cells were resuspended in in $500 \mu 1$ Annexin V binding buffer, and $5 \mu \mathrm{l}$ Annexin V-FITC and $10 \mu \mathrm{l}$ PI were added; cells were incubated for $15 \mathrm{~min}$ at room temperature in the dark and analyzed by flow cytometry (Beckman Coulter, Inc., Brea CA, USA) with Cell Quest software v5.1 (BD Biosciences). Non-viable cells were stained only by PI (Q1); live cells possessed no staining of PI or Annexin V-FITC (Q2); early apoptotic cells exhibited a high degree of Annexin V-FITC staining without PI staining (Q3); and late apoptotic cells had a high degree of PI and Annexin V-FITC staining (Q4). The apoptotic rate was calculated as: $[(\mathrm{Q} 3+\mathrm{Q} 4) /(\mathrm{Q} 1+\mathrm{Q} 2+\mathrm{Q} 3+\mathrm{Q} 4)] \times$ 100. Each experiment was performed in triplicate.

Statistical analysis. SPSS 17.0 (SPSS, Inc., Chicago, IL, USA) was used to perform statistical analysis. All the experiments were repeated three times independently and data are presented as the mean \pm standard deviation. Statistical significance was compared between the treatment and controls groups using the one-way analysis of variance followed by a 

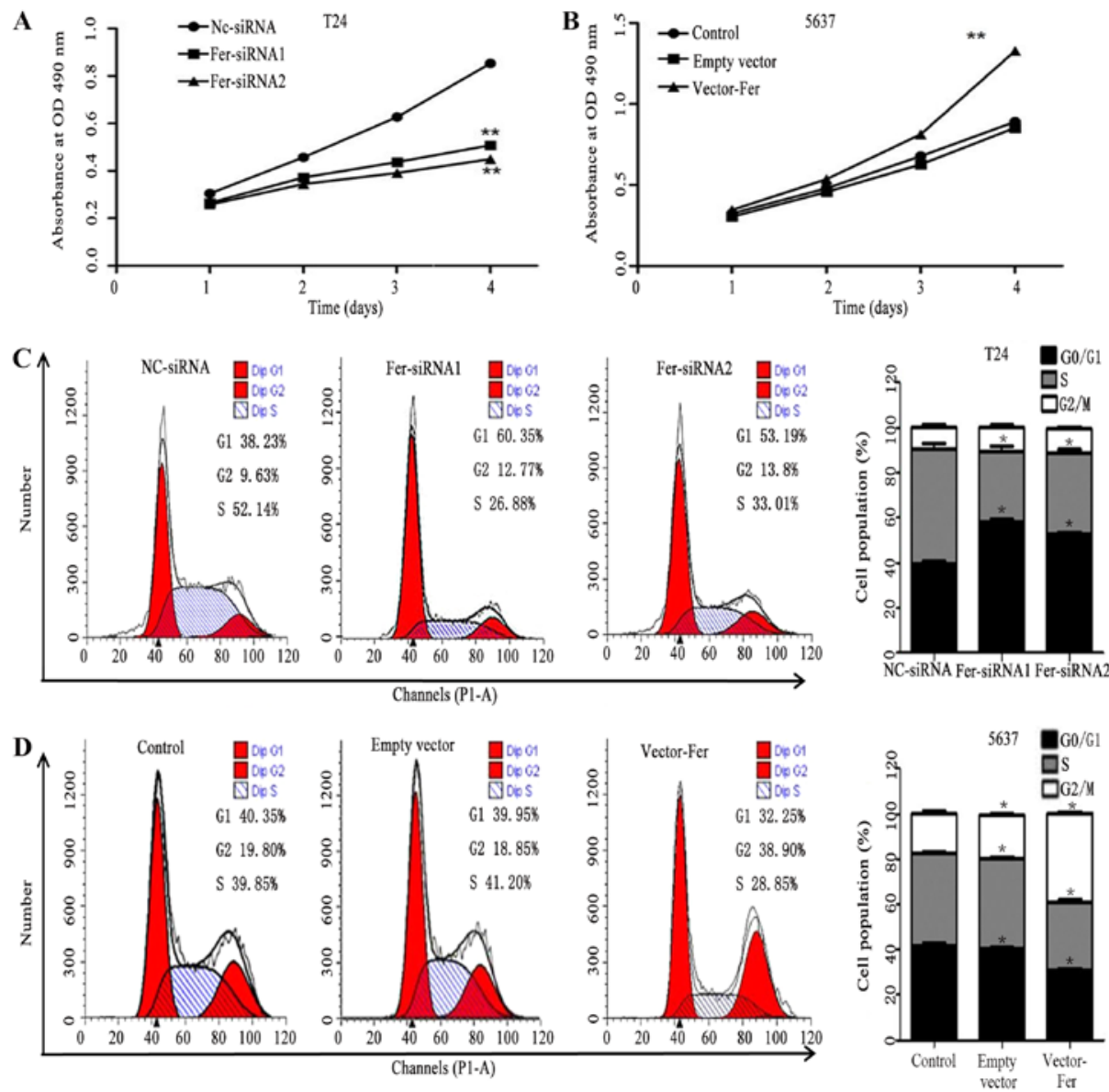

Figure 2. Effects of down- and upregulation of Fer on cell viability and the cell cycle. (A) T24 cells transfected with Fer siRNA exhibited reduced viability compared with NC-siRNA group cells as detected by an MTT assay. The bars represent the mean \pm standard deviation; $n=3 ;{ }^{* *} \mathrm{P}<0.01$ vs. NC-siRNA. (B) Fer overexpression by plasmid transfection significantly promoted the proliferation ability of 5637 cells compared with the control groups. The bars represent the mean \pm standard deviation; $n=3 ;{ }^{* * *} \mathrm{P}<0.01$ vs. Empty vector and Control. (C) Cell cycle of $\mathrm{T} 24$ cells was examined by flow cytometry and downregulation of Fer resulted in an increase in G0/G1 cells, which was accompanied with a reduction of S phase. (D) Fer overexpression in 5637 cells induced a decrease in the proportion of cells in G0/G1 and S phase, and an increase in G2/M cells. " $\mathrm{P}<0.05$ vs. Empty vector and Control. All experiments were repeated three times. Fer, feline sarcoma-related protein; OD, optical density; NC, negative control; siRNA, small interfering RNA; Vector-Fer, Fer overexpression plasmid.

Student-Newman-Keuls test. $\mathrm{P}<0.05$ was considered to indicate a statistically significant difference.

\section{Results}

Fer expression in bladder cancer cell lines. The mRNA and protein expression levels of Fer in SV-HUC-1, 5637 and T24 cells were examined by RT-qPCR and western blot analysis, respectively. The expression levels of Fer mRNA and protein were significantly upregulated in the two bladder cancer cell lines (5637 and T24) compared with expression in the SV-HUC-1 normal bladder epithelium cell line (Fig. 1A and B; $\mathrm{P}<0.05)$. Furthermore, the expression of Fer in the highly invasive and mesenchymal-like bladder cancer T24 cells was significantly higher compared with in 5637 cells, and these two bladder cancer cell lines were used in subsequent experiments to determine the biological roles of Fer.

Efficacy of siRNA and overexpression plasmid transfection of Fer in bladder cancer cells. To investigate the biological function of Fer in the progression of bladder cancer, T24 cells were transfected with one of three siRNAs against Fer (siRNA1, siRNA2 or siRNA3), whereas 5637 cells were transfected with a Fer overexpression plasmid; Nc-siRNA and empty vectors were used as the respective negative controls. RT-qPCR results demonstrated that the relative Fer mRNA expression levels in the Fer-siRNA1 and Fer-siRNA2 groups were significantly decreased compared with the NC-siRNA group $(\mathrm{P}<0.01$; Fig. $1 \mathrm{C})$; similar results were observed for Fer protein levels $(\mathrm{P}<0.01$; Fig. 1D). However, no significant differences in expression levels were observed in the Fer-siRNA3-transfected cells compared with the NC-siRNA group. Conversely, Fer overexpression in 5637 cells by plasmid transfection. As demonstrated by RT-qPCR and western blot analysis, the expression of Fer was significantly increased compared with the untreated control and the empty vector group $(\mathrm{P}<0.05$; Fig. $1 \mathrm{E}$ and $\mathrm{F})$.

Effects of down- or upregulation of Fer on cell viability and the cell cycle. To investigate the role of Fer on the viability of bladder cancer cells, the viability of T24 and 5637 cells transfected with Fer-siRNAs or Vector-Fer was determined by an MTT assay at days 1, 2, 3 and 4 post-transfection. The results demonstrated that the viability of Fer-siRNA-transfected T24 cells was significantly decreased compared with that of Nc-siRNA-transfected cells $(\mathrm{P}<0.01$; Fig. $2 \mathrm{~A})$; the viability of 
A

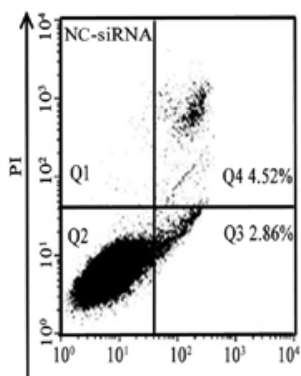

B

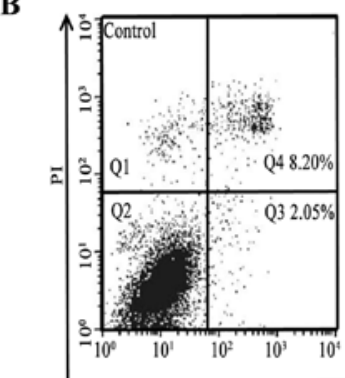

C

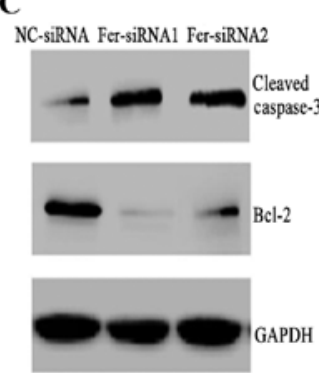

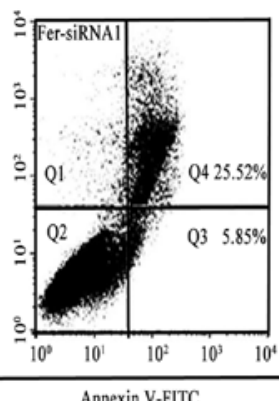
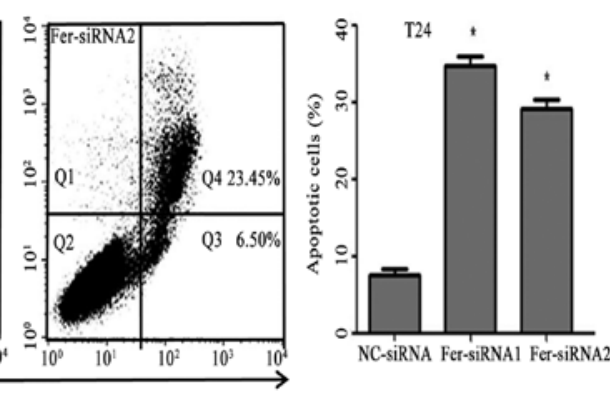

Annexin Y-FITC
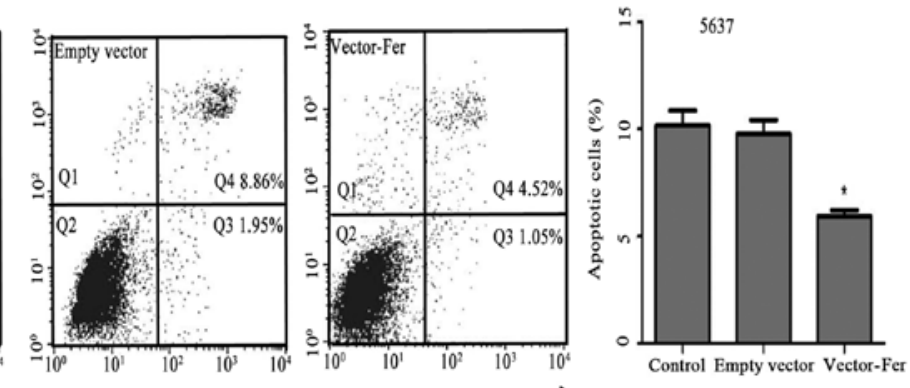

D

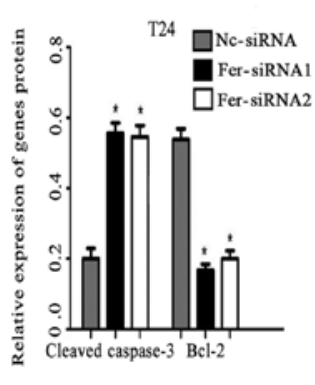

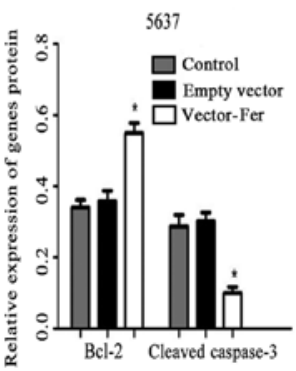

Figure 3. Effects of down- and upregulation of Fer on cell apoptosis. (A) Flow cytometric analysis of apoptosis following staining with PI/Annexin V-FITC indicated that the proportion of apoptotic cells in the Fer-siRNA group of T24 cells was significantly higher compared with in the Nc-siRNA group. The bars represent the mean \pm standard deviation; $\mathrm{n}=3$; ${ }^{*} \mathrm{P}<0.05$ vs. NC-siRNA. (B) Cell apoptosis of 5637 cells overexpressing Fer was investigated by flow cytometry. The proportion of apoptotic cells in the Vector-Fer group was significantly lower compared with in the control group. The bars represent the mean \pm standard deviation; $\mathrm{n}=3 ;{ }^{*} \mathrm{P}<0.05$ vs. Empty vector and Control. (C) Western blot analysis revealed that knockdown of Fer decreased the expression of Bcl-2 and increased that of cleaved caspase-3 in T24 cells. (D) Western blot analysis revealed that overexpression of Fer increased the expression of Bcl-2 and decreased that of cleaved caspase- 3 in 5637 cells. GAPDH was used as a loading control. All experiments were repeated three times; $\mathrm{n}=3$; ${ }^{*} \mathrm{P}<0.05$ vs. NC-siRNA, Empty vector and/or Control. Bcl-2, B-cell lymphoma 2; Fer, feline sarcoma-related protein; FITC, fluorescein isothiocyanate; PI, propidium iodide; NC, negative control; siRNA, small interfering RNA; Vector-Fer, Fer overexpression plasmid.

Vector-Fer-transfected 5637 cells was significantly increased compared with that of the control and empty vector groups at day 4 ( $\mathrm{P}<0.01$; Fig. 2B). Furthermore, flow cytometric analysis was conducted to determine the potential mechanism underlying the effects of Fer on bladder cancer cell viability and cell cycle following Fer knockdown and overexpression. As presented in Fig. 2C and D, Fer-siRNA significantly increased the proportion of cells in G0/G1 phase and reduced the proportion in S phase compared with Nc-siRNA-transfected cells. Conversely, treatment with Vector-Fer significantly reduced the proportion of cells in G0/G1 phase and elevated the number of cells in G2/M phase compared with the controls. Furthermore, the proportion of Vector-Fer-transfected cells at $S$ phase was significantly decreased compared with the control and empty vector groups $(\mathrm{P}<0.05)$. These results indicated that Fer may be closely associated with cell viability and the cell cycle in bladder cancer cells.

Effects of down- or upregulation of Fer on cell apoptosis. To further study the effects of Fer on the apoptosis of bladder cancer cells following transfection an Annexin V/FITC kit and flow cytometry were employed. As presented in Fig. 3A and B, the apoptotic rate of T24 cells in the Fer-siRNA groups was significantly higher compared with the Nc-siRNA group $(\mathrm{P}<0.05)$. However, the apoptotic rate of the Vector-Fer group of 5637 cells was significantly lower compared with the control and empty vector groups $(\mathrm{P}<0.05)$. The results indicated that downregulation of Fer promoted the apoptosis of T24 cells, while overexpression of Fer inhibited 5637 cell apoptosis.

Bcl-2 family proteins regulate cell apoptosis through promoters or inhibitors (15). Cleaved caspase-3, as a prognostic predictor involved in the 'execution' phase of apoptosis, is a key regulator of promoting tumor repopulation induced by dying cells (16). Therefore, the expression of Bcl-2 and cleaved caspase-3, key mediators of apoptosis, was investigated. As presented in Fig. 3C and D, downregulated Fer was associated with significantly increased cleaved caspase-3 expression, whereas the expression of $\mathrm{Bcl}-2$ was significantly downregulated compared with the Nc-siRNA group. On the contrary, Fer overexpression significantly increased $\mathrm{Bcl}-2$ expression 

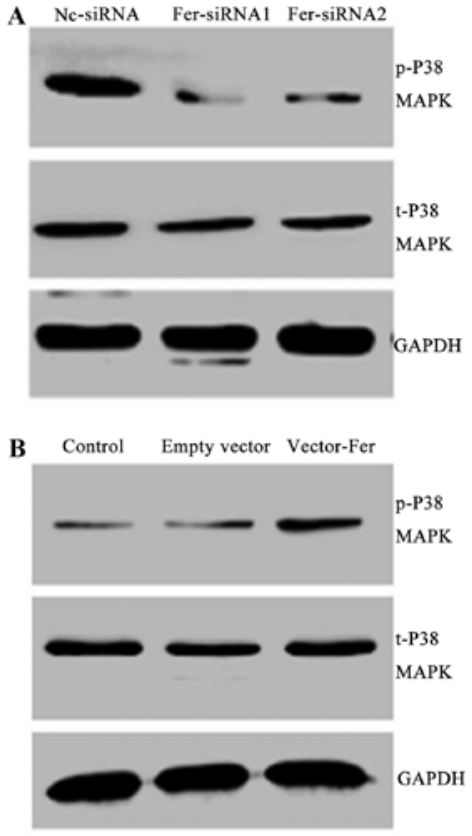

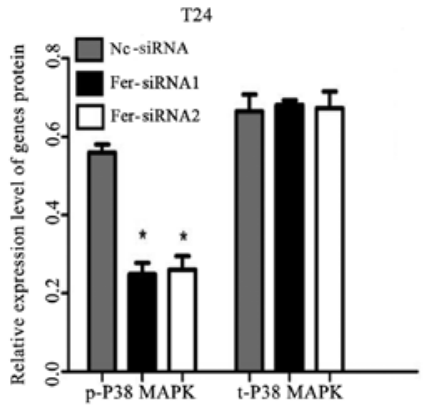

5637

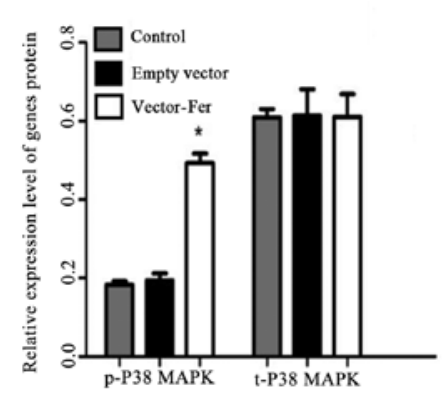

Figure 4. Effects of Fer down- and upregulation on phosphorylation of P38 MAPK. (A) Western blot analysis revealed that knockdown of Fer decreased the expression of p-P38 MAPK in T24 cells. (B) Western blot analysis revealed that Fer overexpression increased the expression of p-P38 MAPK in 5637 cells. GAPDH was used as a loading control. All experiments were repeated three times; $n=3$; ${ }^{\mathrm{P}}<0.05$ vs. NC-siRNA, or Empty vector and Control. Fer, feline sarcoma-related protein; NC, negative control; MAPK, mitogen-activated protein kinase; $p$, phosphorylated; siRNA, small interfering RNA; $t$, total; Vector-Fer, Fer overexpression plasmid.

and downregulated that of cleaved capsase- 2 compared with the controls.

Effects of down- or upregulation of Fer on P38 MAPK phosphorylation. P38 MAPK signaling is one of the main pathways undertaken by MAPK, and serves a key role in regulating cell apoptosis, growth, differentiation and oncogenic transformation (17). Therefore, the effects of Fer down- or upregulation on the P38 MAPK signaling pathway were investigated. As presented in Fig. 4A and B, a significant decrease in the expression of p-P38 MAPK following Fer knockdown in T24 cells compared with in the Nc-siRNA group was observed. Conversely, overexpression of Fer significantly upregulated the expression of p-P38 MAPK in 5637 cells compared with the controls; however, the levels of total P38 MAPK were markedly unaffected in either bladder cancer cell group. These findings suggested that Fer may regulate cell proliferation and apoptosis via P38 MAPK modulation.

\section{Discussion}

Previously, several studies have reported that Fer is widely expressed in proliferating mammalian cells $(18,19)$. Overexpression of Fer has also been associated with poor prognosis in various types of human cancer and serves as a prognostic marker (5,7-9); however, the biological role and underlying mechanism of Fer in bladder cancer cell viability and apoptosis require further investigation. In the present study, Fer expression in normal and bladder cancer cells was analyzed by RT-qPCR and western blotting. The findings revealed that Fer was significantly overexpressed in bladder cancer cells compared with in normal bladder cells. This was consistent with our previous findings in bladder cancer tissues (9). These results indicate that Fer may be involved in the progression of bladder cancer.

A recent report has indicated that Fer activation is required in tumorigenesis and the invasiveness of certain cancer cells in which C-Src is upregulated (20); however, the molecular mechanisms of Fer remain unknown. In the present study, to reveal the biological effects of Fer on cell viability and apoptosis in bladder cancer, the transfection of bladder cancer cells with siRNA or plasmid was conducted to knock down or overexpress Fer, respectively. The effects of downregulation or upregulation of Fer on bladder cancer cell viability and apoptosis were determined. The results revealed that Fer siRNA suppressed the viability and G1/S transition of T24 cells. This resulted in cell cycle arrest at G1, which may explain the inhibition of cell growth induced by Fer knockdown in T24 cells (21). In addition, Annexin FITC/PI staining was used to observe the rate of apoptosis; cell death is the process by which aged or damaged cells are eliminated and serves a key role in carcinogenesis (22). The present study reported that compared with Nc-siRNA-transfected cells, the fluorescence intensity of Fer-siRNA cells increased significantly, indicating that Fer knockdown could induce the apoptosis of T24 bladder cancer cells. Overexpression of Fer in 5637 cells exhibited opposing effects on cell viability, cell cycle and apoptosis in vitro. Bcl-2 is a member of the regulatory Bcl-2 protein family (15). Overexpression of Bcl-2 is associated with cell cycle arrest (23), and the inhibitory effects of Bcl-2 could increase the rate of apoptosis $(24,25)$. Bcl-2 also increases the expression levels of activated caspase- 3 (cleaved caspase-3), which is a key mediator of programmed cell death (16). Therefore, the present study also investigated the association between the activity of Fer, and the expression of the Bcl-2 and caspase-3. Fer knockdown downregulated 
Bcl-2 and upregulated cleaved caspase-3 expression in T24 cells. The overexpression of Fer in 5637 cells had opposing effects, which may explain the inhibition of the cell cycle and apoptosis. Therefore, these findings indicate that Fer serves an important role in the biological behavior of T24 cells, which is mediated by regulating the expression of certain genes.

Additionally, present study reported that knockdown or overexpression of Fer disrupted the MAPK signaling pathway by altering the expression of phosphorylated p38 MAPK, whereas the expression of total p38 MAPK protein did not notably change. Senis et al (26) revealed that many small G protein/MAPK cascades are involved in downstream signal transduction of FPS/FES tyrosine kinase. Craig and Greer (27) also revealed that Fer kinase is required for sustained p38 kinase activation and the maximal chemotaxis of activated mast cells. Of note, p38 MAPK is activated via sequential phosphorylation in the MAPK signaling pathway (28). Therefore, the results of the present study suggest that Fer may affect cell viability and apoptosis via the P38 MAPK signaling pathway.

In conclusion, these findings contribute to the increasing evidence that Fer is involved in the development and progression of cancer. To the best of our knowledge, the present study is the first to report of Fer as a novel regulator of cell viability and apoptosis in bladder cancer. Fer was indicated to exert its effects by regulating the expression of certain genes and inhibiting the p38 MAPK signaling pathway. In addition, the association between Fer, and viability and apoptosis of bladder cancer cells was determined. Future investigation into the association between phosphorylated P38 and apoptosis is under way. Collectively, the results of the present study indicated that Fer serves a role in the biological behavior of bladder cancer cells, and suggest that Fer may be considered as a novel molecular target for the treatment of bladder cancer.

\section{Acknowledgements}

Not applicable.

\section{Funding}

This study was supported by grants from The National Natural Science Foundation of China (grant nos. 81373005, 81072330 and 81202194) and by The Priority Academic Program Development of Jiangsu Higher Education Institutions.

\section{Availability of data and materials}

The datasets used and/or analyzed during the current study are available from the corresponding author on reasonable request.

\section{Authors' contributions}

XH, DY and CZ conceived and designed the study. ZG, JX and $\mathrm{XH}$ performed cell culture and cell transfection. XM and $\mathrm{XH}$ performed reverse transcription-quantitative polymerase chain reaction, western blotting, MTT assay and flow cytometry. MB, FJ and $\mathrm{XH}$ provided reagents and interpreted the data. XH performed data analysis and wrote the manuscript. All authors read and approved the final manuscript.

\section{Ethics approval and consent to participate}

Not applicable.

\section{Patient consent for publication}

Not applicable.

\section{Competing interests}

The authors declare that they have no competing interests.

\section{References}

1. Babjuk M, Oosterlinck W, Sylvester R, Kaasinen E, Böhle A, Palou-Redorta J and Rouprêt M; Asociación Europea de Urología: EAU guidelines on non-muscle-invasive urothelial carcinoma of the bladder, the 2011 update. Actas Urol Esp 36: 389-402, 2012 (In Spanish).

2. Stenzl A, Cowan NC, De Santis M, Jakse G, Kuczyk MA Merseburger AS, Ribal MJ, Sherif A and Witjes JA: The updated EAU guidelines on muscle-invasive and metastatic bladder cancer. Eur Urol 55: 815-825, 2009.

3. DeGraff DJ, Clark PE, Cates JM, Yamashita H, Robinson VL, Yu X, Smolkin ME, Chang SS, Cookson MS, Herrick MK, et al: Loss of the urothelial differentiation marker FOXA1 is associated with high grade, late stage bladder cancer and increased tumor proliferation. PLoS One 7: e36669, 2012.

4. Greer P: Closing in on the biological functions of Fps/Fes and Fer. Nat Rev Mol Cell Biol 3: 278-289, 2002.

5. Ahn J, Truesdell P, Meens J, Kadish C, Yang X, Boag AH and Craig AW: Fer protein-tyrosine kinase promotes lung adenocarcinoma cell invasion and tumor metastasis. Mol Cancer Res 11: 952-963, 2013.

6. Li H, Ren Z, Kang X, Zhang L, Li X, Wang Y, Xue T, Shen Y and Liu Y: Identification of tyrosine-phosphorylated proteins associated with metastasis and functional analysis of FER in human hepatocellular carcinoma cells. BMC Cancer 9: 366, 2009.

7. Rocha J,Zouanat FZ, Zoubeidi A, Hamel L, Benidir T, Scarlata E, Brimo F, Aprikian A and Chevalier S: The Fer tyrosine kinase acts as a downstream interleukin- 6 effector of androgen receptor activation in prostate cancer. Mol Cell Endocrinol 381: 140-149, 2013.

8. Albeck JG and Brugge JS: Uncovering a tumor suppressor for triple-negative breast cancers. Cell 144: 638-640, 2011.

9. Hu X, Zhang Z, Liang Z, Xie D, Zhang T, Yu D and Zhong C: Downregulation of feline sarcoma-related protein inhibits cell migration, invasion and epithelial-mesenchymal transition via the ERK/AP-1 pathway in bladder urothelial cell carcinoma. Oncol Lett 13: 686-694, 2017.

10. Allard P, Zoubeidi A, Nguyen LT, Tessier S, Tanguay S, Chevrette M, Aprikian A and Chevalier S: Links between Fer tyrosine kinase expression levels and prostate cell proliferation. Mol Cell Endocrinol 159: 63-77, 2000.

11. Pasder O, Shpungin S, Salem Y, Makovsky A, Vilchick S, Michaeli S, Malovani $\mathrm{H}$ and Nir U: Downregulation of Fer induces PP1 activation and cell-cycle arrest in malignant cells. Oncogene 25: 4194-4206, 2006.

12. Xu G, Craig AW, Greer P, Miller M, Anastasiadis PZ, Lilien J and Balsamo J: Continuous association of cadherin with beta-catenin requires the non-receptor tyrosine-kinase Fer. J Cell Sci 117: 3207-3219, 2004

13. Sangrar W, Gao Y, Scott M, Truesdell P and Greer PA: Fer-mediated cortactin phosphorylation is associated with efficient fibroblast migration and is dependent on reactive oxygen species generation during integrin-mediated cell adhesion. Mol Cell Biol 27: 6140-6152, 2007.

14. Livak KJ and Schmittgen TD: Analysis of relative gene expression data using real-time quantitative PCR and the 2(-Delta Delta C(T)) Method. Methods 25: 402-408, 2001.

15. Burlacu A: Regulation of apoptosis by Bcl-2 family proteins. J Cell Mol Med 7: 249-257, 2003.

16. Alnemri ES, Livingston DJ, Nicholson DW, Salvesen G, Thornberry NA, Wong WW and Yuan J: Human ICE/CED-3 protease nomenclature. Cell 87: 171, 1996. 
17. Cuadrado A and Nebreda AR: Mechanisms and functions of $\mathrm{p} 38$ MAPK signalling. Biochem J 429: 403-417, 2010.

18. Letwin K, Yee SP and Pawson T: Novel protein-tyrosine kinase cDNAs related to fps/fes and eph cloned using anti-phosphotyrosine antibody. Oncogene 3: 621-627, 1988.

19. Hao QL, Heisterkamp N and Groffen J: Isolation and sequence analysis of a novel human tyrosine kinase gene. Mol Cell Biol 9: $1587-1593,1989$

20. Oneyama C, Yoshikawa Y, Ninomiya Y, Iino T, Tsukita S and Okada M: Fer tyrosine kinase oligomer mediates and amplifies Src-induced tumor progression. Oncogene 35: 501-512, 2016.

21. Wang Y, Wang T, Han Y, Wu H, Zhao W, Tong D, Wei L, Zhong Z, An R and Wang Y: Reduced ING4 Expression Is Associated with the Malignancy of Human Bladder. Urol Int 94: 464-471, 2015.

22. Kelekar A and Thompson CB: Bcl-2-family proteins: The role of the BH3 domain in apoptosis. Trends Cell Biol 8: 324-330, 1998

23. Simpson NH, Singh RP, Emery AN and Al-Rubeai M: Bcl-2 over-expression reduces growth rate and prolongs G1 phase in continuous chemostat cultures of hybridoma cells. Biotechnol Bioeng 64: 174-186, 1999.
24. Cleary ML, Smith SD and Sklar J: Cloning and structural analysis of cDNAs for bcl-2 and a hybrid bcl-2/immunoglobulin transcript resulting from the $\mathrm{t}(14 ; 18)$ translocation. Cell 47: 19-28, 1986.

25. Yang L, Wu X, Wang D, Luo C and Chen L: Renal carcinoma cell-derived exosomes induce human immortalized line of Jurkat T lymphocyte apoptosis in vitro. Urol Int 91: 363-369, 2013.

26. Senis YA, Sangrar W, Zirngibl RA, Craig AW, Lee DH and Greer PA: Fps/Fes and Fer non-receptor protein-tyrosine kinases regulate collagen- and ADP-induced platelet aggregation. J Thromb Haemost 1: 1062-1070, 2003.

27. Craig AW and Greer PA: Fer kinase is required for sustained p38 kinase activation and maximal chemotaxis of activated mast cells. Mol Cell Biol 22: 6363-6374, 2002.

28. Wada T and Penninger JM: Mitogen-activated protein kinases in apoptosis regulation. Oncogene 23: 2838-2849, 2004. 